\title{
c-Myc promotes cholangiocarcinoma cells to overcome contact inhibition via the mTOR pathway
}

\author{
GUOSONG LUO ${ }^{1,2^{*}}$, BIN LI $^{2 *}$, CHUNYAN DUAN $^{3 *}$, YING CHENG $^{3}$, BIN XIAO $^{3}$, \\ FULI YAO $^{3}$, MEI WEI $^{4}$, QINGHUA TAO ${ }^{5}$, CHUNHONG FENG $^{1}$, XIANMING XIA $^{1}$, \\ HONG ZHOU $^{3}$, XIAOFANG ZHAO ${ }^{2,3}$ and RONGYANG DAI ${ }^{2,4}$ \\ ${ }^{1}$ Department of Hepatobiliary Surgery of the Affiliated Hospital, ${ }^{2}$ Liver Diseases Laboratory, \\ ${ }^{3}$ Department of Biochemistry and Molecular Biology, ${ }^{4}$ Department of Liver Diseases \\ of the Affiliated Hospital of Chinese Traditional Medicine, Southwest Medical University, \\ Luzhou, Sichuan 646000; ${ }^{5}$ MOE Key Laboratory of Protein Sciences, \\ Tsinghua University School of Life Sciences, Beijing 100084, P.R. China
}

Received March 19, 2017; Accepted August 14, 2017

DOI: $10.3892 / o r .2017 .5913$

\begin{abstract}
The loss of contact inhibition is a hallmark of a wide range of human cancer cells. Yet, the precise mechanism behind this process is not fully understood. c-Myc plays a pivotal role in carcinogenesis, but its involvement in regulating contact inhibition has not been explored to date. Here, we report that c-Myc plays an important role in abrogating contact inhibition in human cholangiocarcinoma (CCA) cells. Our data show that the protein level of c-Myc obviously decreased in contact-inhibited normal biliary epithelial cells. However, CCA cells sustain high protein levels of c-Myc and keep strong proliferation ability in confluent conditions. Importantly, the suppression of c-Myc by inhibitor or siRNA induced G0/G1 phase cell cycle arrest in confluent CCA cells. We demonstrate that the inhibition of c-Myc suppressed the activity of mammalian target of rapamycin (mTOR) in confluent CCA cells, and mTOR inhibition induced G0/G1 phase cell cycle arrest in confluent CCA cells. In confluent CCA cells, the activity of Merlin is downregulated, and Yes-associated protein (YAP) sustains high levels of activity. Furthermore, YAP inhibition not only induced G0/G1 phase cell cycle arrest, but also decreased c-Myc expression in confluent CCA cells. These results indicate that Merlin/YAP/c-Myc/mTOR signaling axis promotes human CCA cell proliferation by overriding contact
\end{abstract}

Correspondence to: Professor Rongyang Dai or Dr Xiaofang Zhao, Liver Diseases Laboratory, Southwest Medical University, Luzhou, Sichuan 646000, P.R. China

E-mail: dryrun2502@163.com

E-mail: zhaoxiaofang2099@163.com

*Contributed equally

Key words: cholangiocarcinoma, contact inhibition, c-Myc, mTOR inhibition. We propose that overriding c-Myc-mediated contact inhibition is implicated in the development of CCA.

\section{Introduction}

Cell contact inhibition is a critical property of normal cells to stop cell proliferation upon reaching confluence (1). In vitro, non-transformed cells are arrested in G0/G1 phase at high density to form a confluent monolayer. In vivo, contact inhibition keeps homeostasis of cell number and thus maintains normal tissue architecture and the organ size (2). In contrast to normal cells, cancer cells usually exhibit a loss of growth control and escape contact inhibition, enabling them to replicate limitlessly $(3,4)$. There are numbers of oncogenes and tumor suppressor genes reported to regulate contact inhibition, but the precise mechanisms behind the loss of cell contact inhibition of cancer cells remain largely unknown.

The c-myc proto-oncogene encodes a ubiquitously expressed transcription factor which plays a central role in cell growth and proliferation regulating (5). It has been reported that c-Myc is deregulated in nearly half of human tumors, such as hepatocellular carcinoma (HCC) and cholangiocarcinoma (CCA) (6-8). c-Myc contains a basic region and a helix-loop-helix/leucine zipper (HLH/LZ) domain. Through the HLH/LZ domain, c-Myc heterodimerizes with another transcription factor $\operatorname{Max}(9,10)$. The c-Myc/Max complex activates the expression of target genes through binding enhancer box sequences (E-boxes) and recruiting histone acetyltransferases (HATs) (11). c-Myc is induced rapidly when cells transit from quiescent to proliferative state, and the induction of c-Myc is required for cell growth and proliferation $(12,13)$. It has been reported that forced overexpression of c-Myc can induce cell cycle re-entry in quiescent cells, and the inhibition of c-Myc can lead to the suppression of cell cycle $(14,15)$.

CCA is a highly malignant tumor originating from biliary epithelial cells (16). As usually diagnosed at advanced stage, the prognosis of CCA is very poor. CCA is a heterogeneous 
tumor and the molecular mechanisms underlying the development of CCA are largely unknown. Since c-Myc is induced during cholestatic liver injury and CCA progression, and c-Myc knockdown inhibits the progression of CCA $(17,18)$, it is important to uncover the detailed mechanism of c-Myc in CCA.

Owing to the critical role of c-Myc in cell proliferation regulation, we wonder whether c-Myc participates in the loss of contact inhibition of CCA cells. Here, we find that c-Myc sustains high protein levels in confluent human CCA cells, but not in human normal biliary epithelial cells. The inhibition of c-Myc restores contact inhibition of human CCA cells. Furthermore, we show that c-Myc promotes the loss of contact inhibition in human CCA cells through the mTOR pathway, and the deregulation of c-Myc is controlled by Yes-associated protein (YAP). Our findings indicate that c-Myc is an important promoter mediating the loss of contact inhibition in human CCA cells.

\section{Materials and methods}

Chemicals and antibodies. c-Myc inhibitor 10058-F4 (F4) and YAP inhibitor verteporfin (VP) were purchased from Selleck Chemicals (Houston, TX, USA). mTOR inhibitor rapamycin was purchased from Tocris Bioscience (Bristol, UK). Antibodies against cyclin D1, p27, c-Myc, p-p70S6K, p70S6K, p-S6, p-YAP (ser127), YAP, p-Merlin and Merlin were purchased from Cell Signaling Technology (Danvers, MA, USA). Antibody against GAPDH, human c-Myc siRNA and control siRNA were purchased from Santa Cruz Biotechnology (Heidelberg, Germany).

Cell culture. Human normal biliary epithelial cell line HIBEC and CCA cell lines QBC939 and RBE were cultured in RPMI-1640 medium supplemented with $10 \%$ fetal bovine serum and $1 \%$ penicillin/streptomycin in a humidified incubator containing $5 \% \mathrm{CO}_{2}$ and $95 \%$ ambient air at $37^{\circ} \mathrm{C}$. Cells were plated at different densities to achieve low $(-50 \%$ confluent cells) and high (confluent cells) densities.

Western blot analysis. Cells were lysed in Triton lysis buffer (20 mM Tris, pH 7.4, $137 \mathrm{mM} \mathrm{NaCl,} \mathrm{10 \%} \mathrm{glycerol,}$ $1 \%$ Triton X-100, 2 mM EDTA, 1 mM PMSF, $10 \mathrm{mM} \mathrm{NaF}$, $5 \mathrm{mg} / \mathrm{ml}$ aprotinin, $20 \mathrm{mM}$ leupeptin, and $1 \mathrm{mM}$ sodium orthovanadate) and centrifuged at $12,000 \mathrm{~g}$ for $15 \mathrm{~min}$. Protein concentrations were measured using the BCA assay. Protein samples were denatured with $4 \mathrm{X}$ SDS-loading buffer (200 mM Tris, pH 6.8, 8\% SDS, 400 mM DTT, 0.4\% bromphenol blue, $40 \%$ glycerol) at $100^{\circ} \mathrm{C}$ for $5 \mathrm{~min}$ and subjected to standard SDS-PAGE and western blot analysis as previously described (19). The proteins were electrolytically transferred to an NC membrane and blocked in TBST containing 5\% non-fat milk at room temperature for $1 \mathrm{~h}$. The membrane was incubated at $4^{\circ} \mathrm{C}$ overnight with primary antibodies against cyclin D1 (1:1,000), p27 (1:1,000), c-Myc (1:1,000), p-p70S6K $(1: 1,000)$, p70S6K $(1: 1,000), \mathrm{p}-\mathrm{S} 6(1: 1,000), \mathrm{p}-Y A P$ (ser127) $(1: 1,000)$, YAP $(1: 1,000)$, p-Merlin $(1: 1,000)$, Merlin $(1: 1,000)$ and GAPDH (1:200). Then the membrane was incubated with secondary antibodies labeled with IRDye 700 (Rockland Immunochemicals, Gilbertsville, PA, USA). Protein levels were detected by the Odyssey system (LiCor, Lincoln, NE, USA).

Cell cycle analysis by flow cytometry. Cells were fixed overnight, resuspended in phosphate-buffered saline (PBS), and stained with propidium iodide in the dark for $30 \mathrm{~min}$. The DNA content was measured by fluorescence-activated cell sorting (FACS) on a Becton-Dickinson FACScan flow cytometry system.

RNA interference. Transient transfections were performed with Lipofectamine 2000 (Invitrogen) according to the manufacturer's instructions. Briefly, for each $35-\mathrm{mm}$ dish, $10 \mu \mathrm{l}$ of $20 \mu \mathrm{M}$ stock of duplex was mixed with $100 \mu \mathrm{l}$ of DMEM while in a separate tube $8 \mu \mathrm{l}$ of Lipofectamine 2000 was mixed with $100 \mu \mathrm{l}$ of DMEM. The two solutions were then gently combined and added to prewashed cells, in normal medium including serum. The cultures were then left for $6 \mathrm{~h}$ before washing and refeeding.

Statistical analysis. Results were expressed as the mean \pm SD. Statistical analysis was performed using Student's t-test. $\mathrm{P}<0.05$ was considered statistically significant.

\section{Results}

Human CCA cells are resistant to contact inhibition. To determine the roles of cell density in the proliferation of normal biliary epithelial cells and CCA cells, we plated normal biliary epithelial cells HIBEC and CCA cells QBC939 and RBE at low density and high density. The flow cytometry data showed that contact-inhibited normal biliary epithelial cells HIBEC were arrested in G0/G1 phase (Fig. 1A). It is notable that CCA cells QBC939 and RBE keep strong proliferation ability at high density (Fig. 1A). These data indicated that CCA cells are resistant to contact inhibition. As cyclin D1, which is required for $\mathrm{G} 1 \rightarrow \mathrm{S}$ phase transition, is a key regulator of cell cycle progression (20), we checked the protein levels of cyclin D1 in HIBEC, QBC939 and RBE cells at low and high densities, respectively. Compared with low density, high density obviously decreased the protein level of cyclin D1 in HIBEC cells (Fig. 1B). While, high density had no apparent effect on the protein levels of cyclin D1 in QBC939 and RBE cells (Fig. 1B). p27, a marker of G0/G1 phase arrest, has been shown to play an important role in contact inhibition $(21,22)$. Then, we assessed the protein levels of p27 in HIBEC, QBC939 and RBE cells at low and high densities. As shown in Fig. 1B, contact inhibition induced the expression of p27 in HIBEC cells. However, in QBC939 and RBE cells, there was no apparent difference of $\mathrm{p} 27$ protein levels between low and high densities. These data indicate that CCA cells are resistant to contact inhibition.

c-Myc contributes to contact inhibition loss in human CCA cells. Since c-Myc is a crucial regulator for cell cycle, and its deregulation is implicated in CCA (23), we set out to investigate whether c-Myc is involved in contact inhibition resistance in CCA cells. We evaluated the protein levels of c-Myc in HIBEC, QBC939 and RBE cells at low and high densities. We found that c-Myc protein levels are clearly lower in confluent 
A
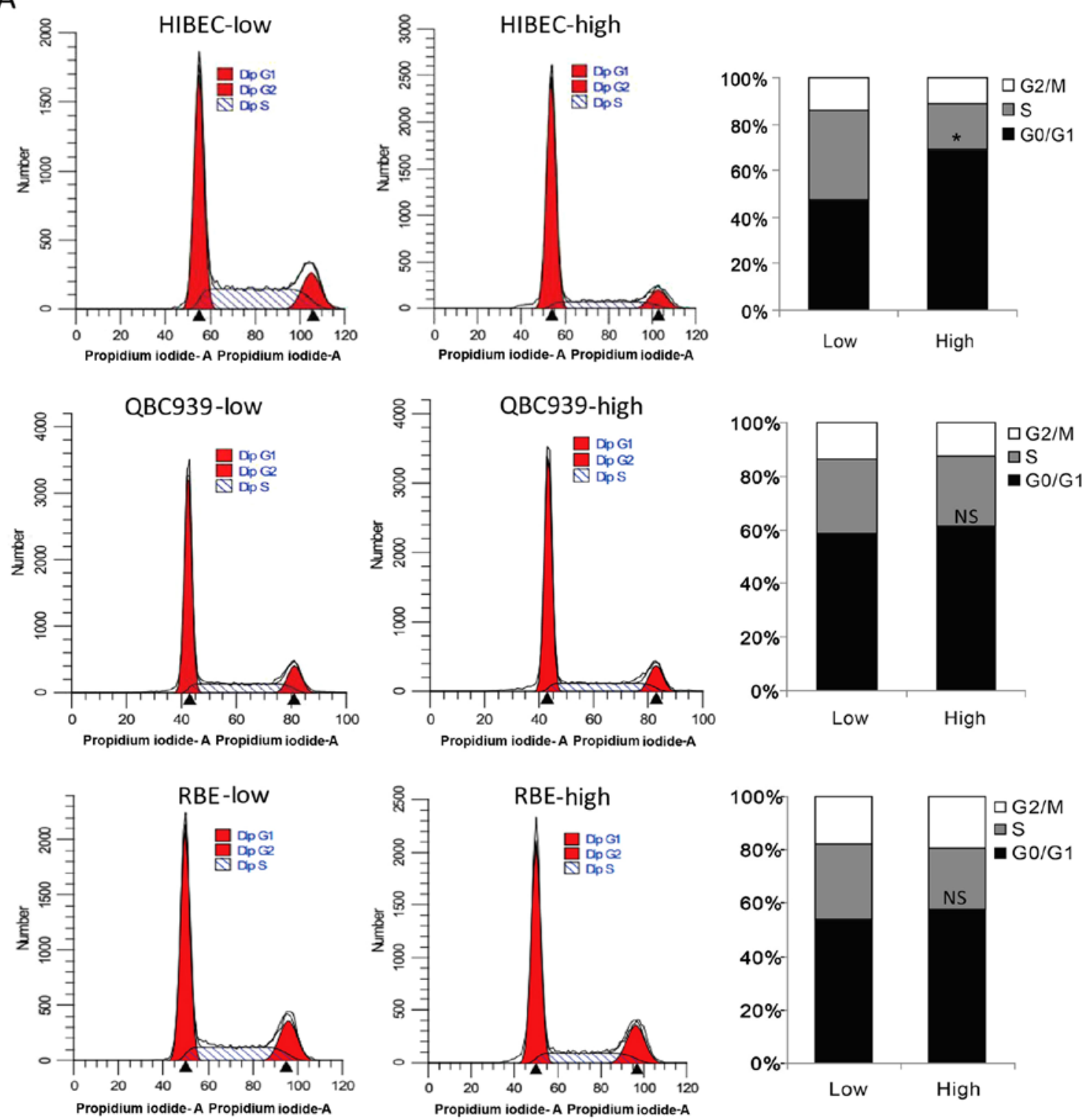

B
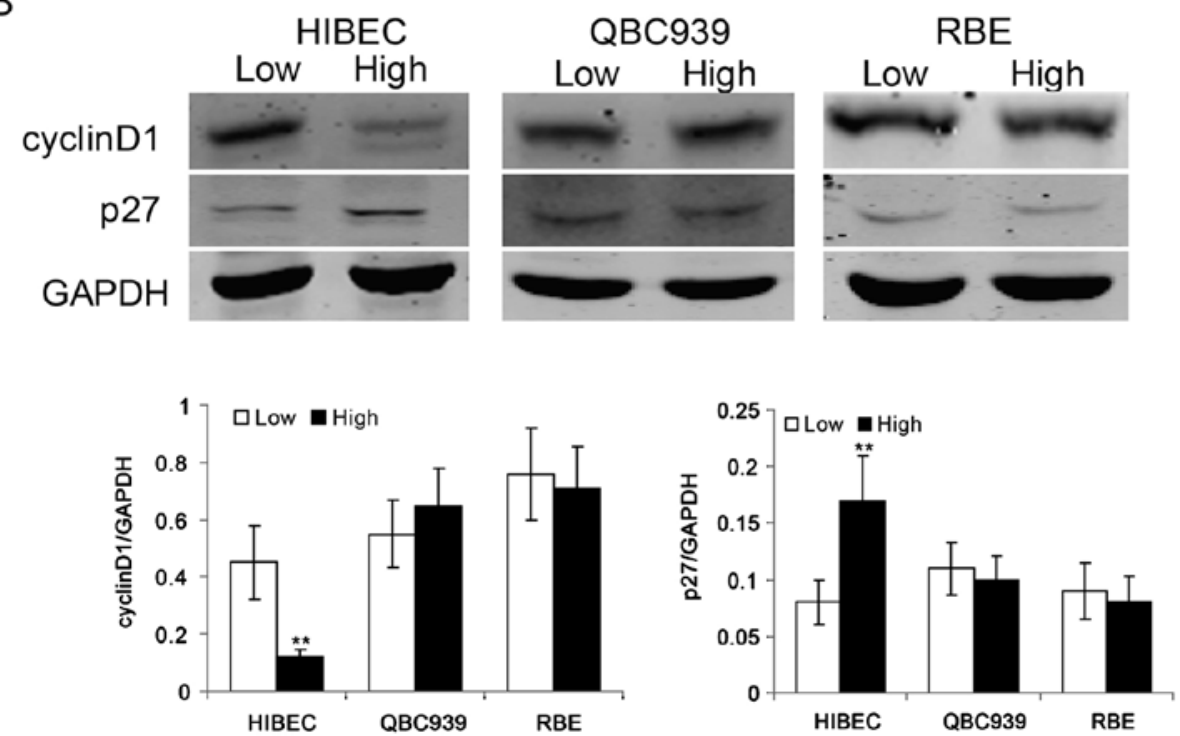

Figure 1. Human CCA cells are resistant to contact inhibition. (A) HIBEC, QBC939 and RBE cells at low and high densities were subjected to measurement of cell cycle distribution using flow cytometry after staining with propidium iodide. Data are representative of three individual experiments. (B) Cell lysates from HIBEC, QBC939 and RBE cells at low and high densities were subjected to western blot analysis of cyclin D1 and p27 expression. GAPDH was used as loading control. The intensities of cyclin D1 and p27 were quantified by Image Studio and normalized to GAPDH. 
A

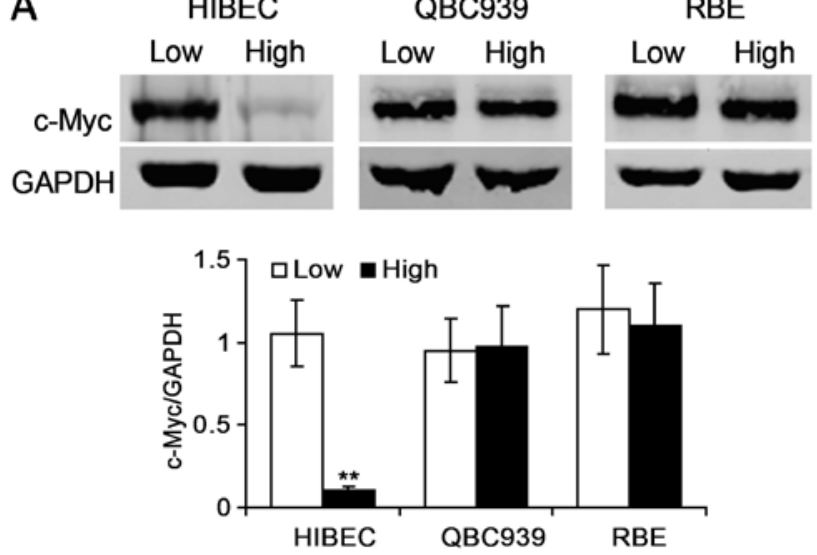

B
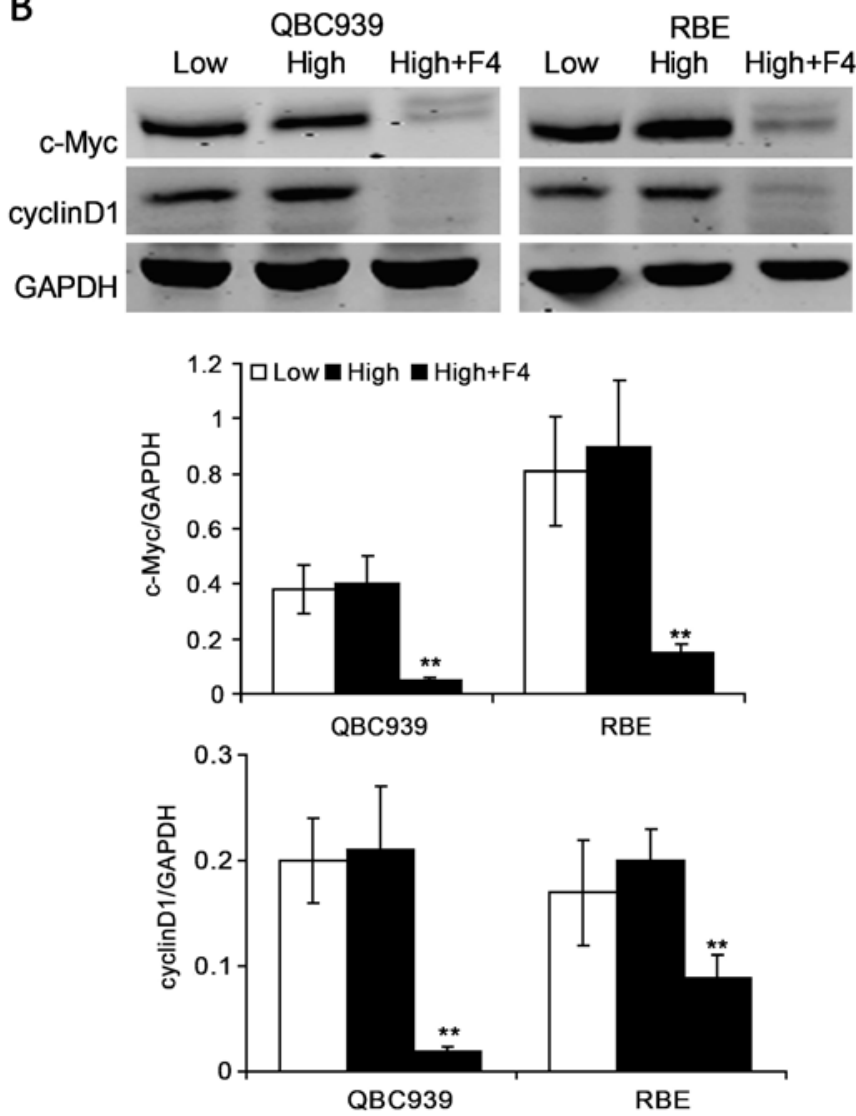

Figure 2. c-Myc sustains high levels of cyclin D1 in confluent human CCA cells. (A) Cell lysates from HIBEC, QBC939 and RBE cells at low and high densities were subjected to western blot analysis of c-Myc expression. GAPDH was used as loading control. The intensities of c-Myc were quantified by Image Studio and normalized to GAPDH. (B) Cell lysates from QBC939 and RBE cells at low and high densities with or without 10058-F4 $(\mathrm{F} 4,100 \mathrm{nM})$ treatment for $24 \mathrm{~h}$ were subjected to western blot analysis of c-Myc and cyclin D1 expression. GAPDH was used as loading control. The intensities of c-Myc and cyclin D1 were quantified by Image Studio and normalized to GAPDH.

HIBEC cells as compared with low density HIBEC cells (Fig. 2A). However, there was no apparent difference in the protein levels of c-Myc between low and high densities in QBC939 and RBE cells (Fig. 2A). c-Myc protein maintained high levels in confluent QBC939 and RBE cells (Fig. 2A). These data indicate that c-Myc may be an important promoter for CCA cells to escape contact inhibition. To determine whether c-Myc leads to contact inhibition resistance in CCA cells, confluent QBC939 and RBE cells were treated with c-Myc specific inhibitor 10058-F4. Our data are consistent with other studies that 10058-F4 effectively suppress the expression of c-Myc (Fig. 2B). Furthermore, the data demonstrated that cyclin D1 clearly decreased upon 10058-F4 treatment in confluent QBC939 and RBE cells (Fig. 2B).

We detected the cell cycle in confluent CCA cells after 10058-F4 treatment. The results showed that 10058-F4 treatment induced G0/G1 phase cell cycle arrest in confluent QBC939 and RBE cells (Fig. 3A). To confirm the role of c-Myc in inhibiting contact inhibition, we used siRNA to knock-down the expression of c-Myc. As shown in Fig. 3B, c-Myc knockdown induced G0/G1 phase cell cycle arrest in confluent QBC939 and RBE cells. These data suggest that $\mathrm{c}-\mathrm{Myc}$ is required for CCA cells to override contact inhibition.

The mTOR pathway is implicated in c-Myc-mediated contact inhibition loss in human CCA cells. How c-Myc induces contact inhibition loss in CCA cells was next investigated. It has been reported that the inactivation of mammalian target of rapamycin (mTOR) is implicated in cell contact inhibition (24). We have previously reported that the mTOR pathway plays an important role in CCA cell growth and survival (25). Here we wonder whether there is a link between c-Myc and mTOR in contact inhibition regulation in CCA cells. We investigated the activity of mTOR in HIBEC and CCA cells at different cell densities. The results showed the levels of phosphorated p70S6K and S6 ribosomal protein (S6), both are markers of the activity of mTOR, decreased obviously in confluent HIBEC cells. In contrast, there is no apparent decrease of phosphor-p70S6K and phosphor-S6 in confluent QBC939 and RBE cells (Fig. 4A). In order to determine whether there is a link between c-Myc and mTOR in confluent CCA cells, we investigated the activity of mTOR in confluent CCA cells upon c-Myc inhibition. The results showed that inhibition of c-Myc by 10058-F4 significantly suppressed the phosphorylation of p70S6K and S6 in confluent QBC939 and RBE cells (Fig. 4B). To further confirm the results, we used siRNA to knock down c-Myc expression. As shown in Fig. 4C, c-Myc knockdown suppressed the phosphorylation of p70S6K and S6 as well as the protein levels of cyclin D1 in confluent QBC939 and RBE cells. These data suggest that the mTOR pathway is involved in c-Myc-induced contact inhibition loss in CCA cells.

To confirm whether mTOR is required for the loss of contact inhibition in CCA cells, we detected cell cycle status in confluent CCA cells after mTOR inhibition. As shown in Fig. 5, mTOR inhibitor rapamycin treatment induced G0/G1 arrest in confluent QBC939 and RBE cells. Together, these data indicate that $\mathrm{c}-\mathrm{Myc} / \mathrm{mTOR}$ deregulation plays a pivotal role in contact inhibition loss in CCA cells.

Deregulated c-Myc in confluent human CCA cells is regulated by YAP signaling. Next, we investigated the underlying mechanism of c-Myc deregulation in confluent CCA cells. As Yes-associated protein (YAP) plays an important role in regulating cell contact inhibition and in cancer development $(26,27)$. We tested whether YAP is involved in c-Myc-mediated contact inhibition loss in CCA cells. The levels of phospho-YAP and total YAP proteins at low and high densities in HIBEC, 

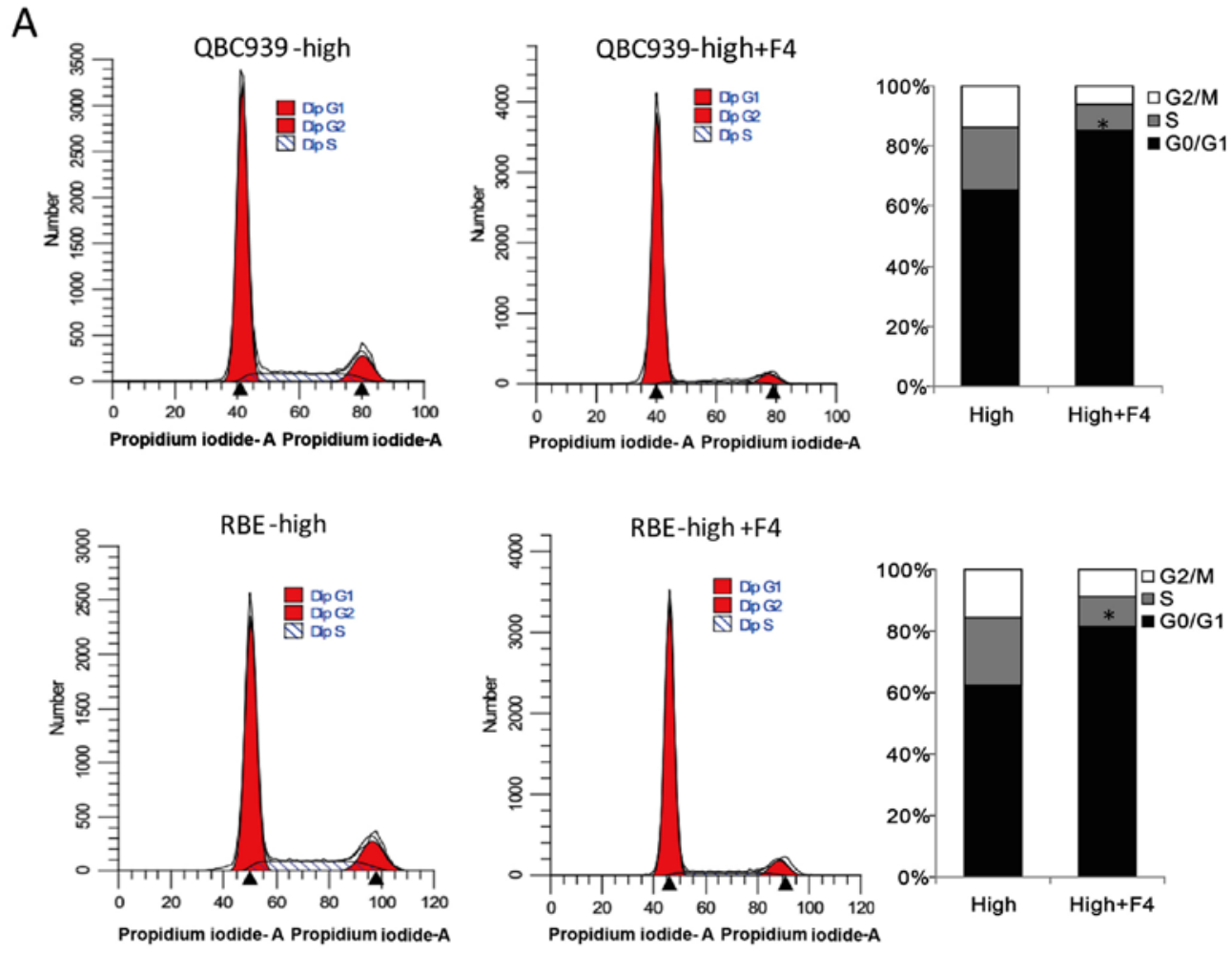

B

QBC939

RBE

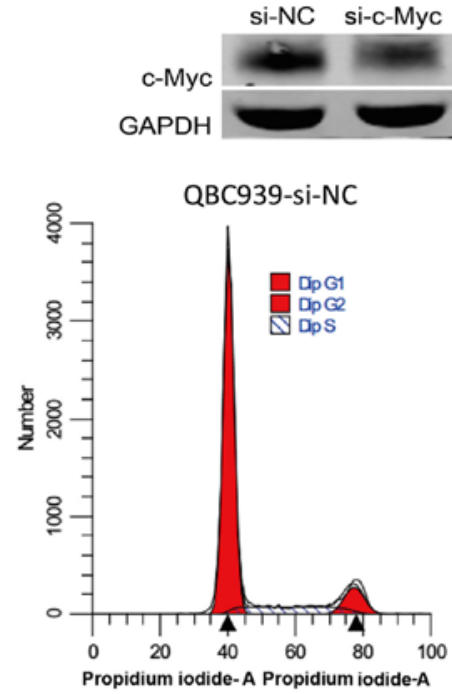

si-NC si-c-Myc
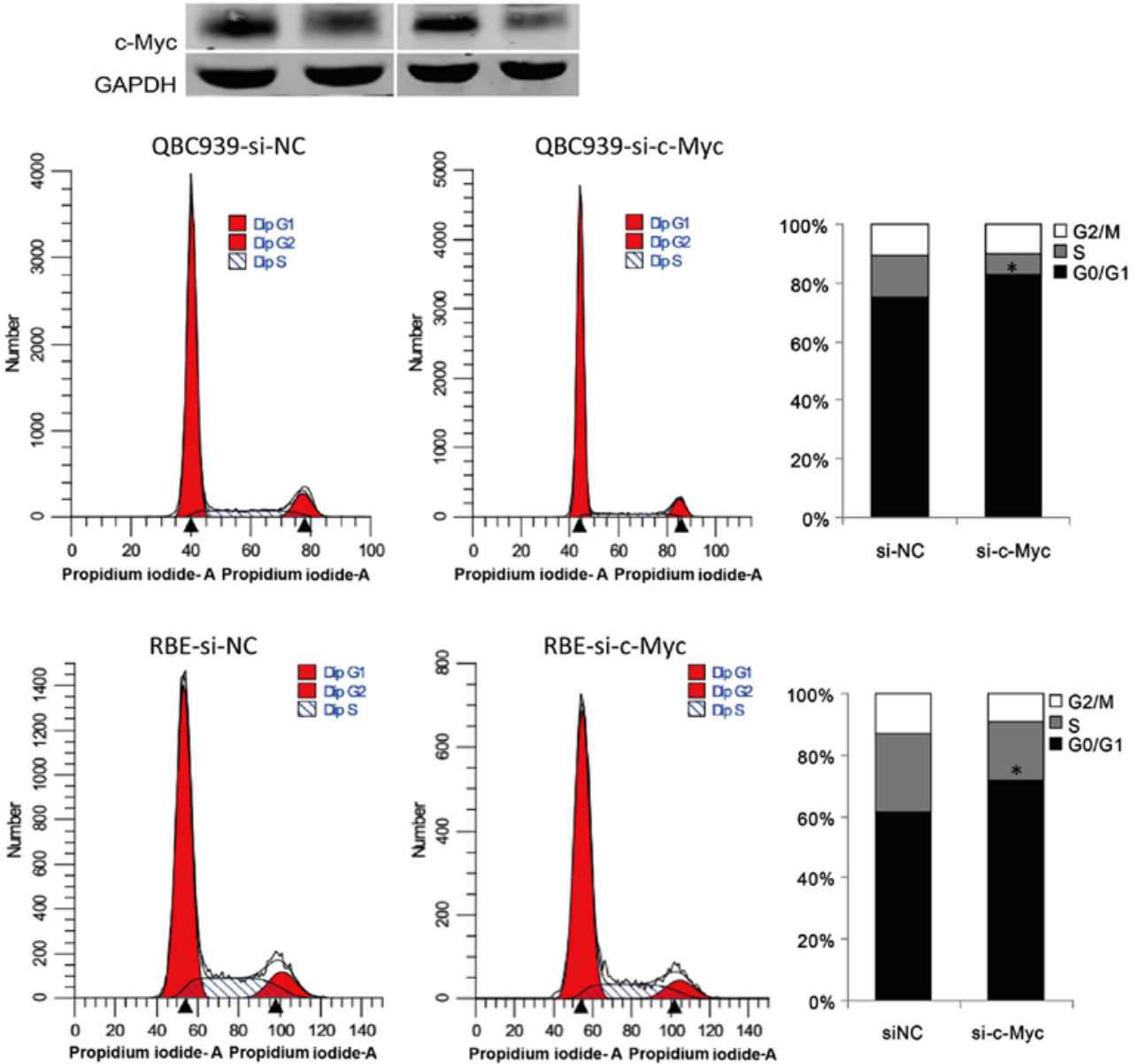

Figure 3. c-Myc contributes to contact inhibition loss in human CCA cells. (A) QBC939 and RBE cells at high density with or without 10058 -F4 (F4, 100 nM) treatment for $24 \mathrm{~h}$ were subjected to measurement of cell cycle distribution using flow cytometry after staining with propidium iodide. (B) Western blot analysis for c-Myc expression in control siRNA (si-NC) or si-c-Myc transfected QBC939 and RBE cells (upper panel). Cell cycle of QBC939 and RBE cells transfected with control si-NC or si-c-Myc were analyzed by flow cytometry (lower panel). Data are representative of three individual experiments. 
A
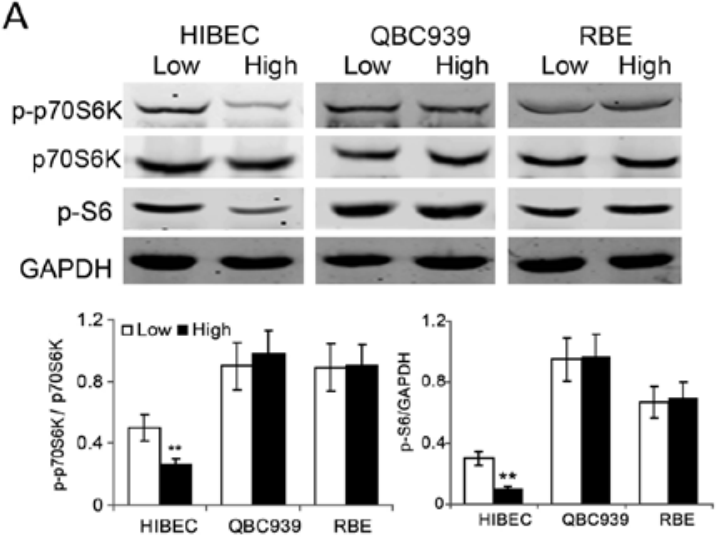

B


C
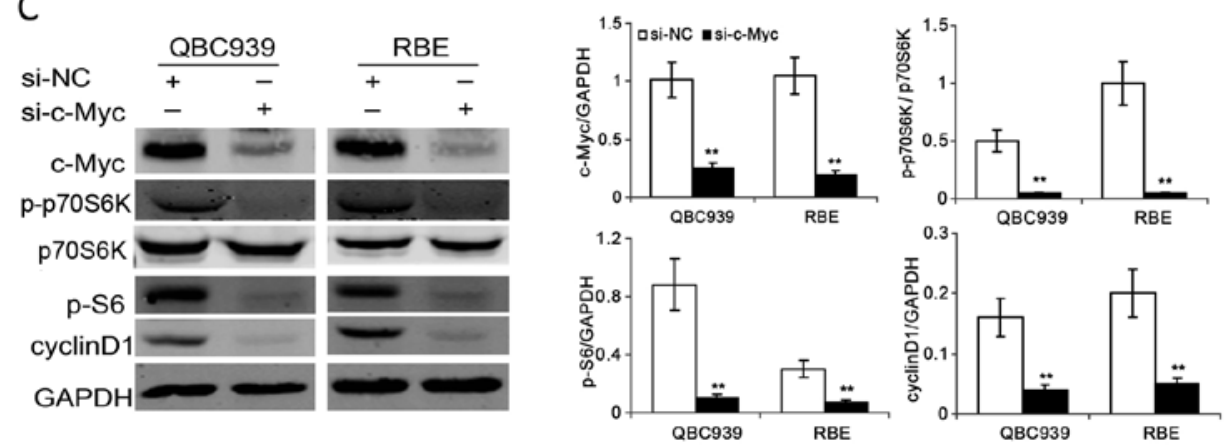

Figure 4. c-Myc sustains the activities of mTOR in confluent human CCA cells. (A) Cell lysates from HIBEC, QBC939 and RBE cells at low and high densities were subjected to western blot analysis of p-p70S6K, p70S6K and p-S6 expression. GAPDH was used as loading control. (B) Cell lysates from QBC939 and RBE cells at low and high densities with or without 10058-F4 (F4, $100 \mathrm{nM})$ treatment for $24 \mathrm{~h}$ were subjected to western blot analysis of p-p70S6K, p70S6K and p-S6 expression. GAPDH was used as loading control. The intensities of p-p70S6K and p-S6 were quantified by Image Studio and normalized to p70S6K or GAPDH. (C) Cell lysates from QBC939 and RBE cells at high density with or without c-Myc siRNA treatment for 36 h were subjected to western blot analysis of p-p70S6K, p70S6K and p-S6 expression. GAPDH was used as loading control. The intensities of c-Myc, p-p70S6K, p-S6 and cyclin D1 were quantified by Image Studio and normalized to p70S6K or GAPDH.

A


B
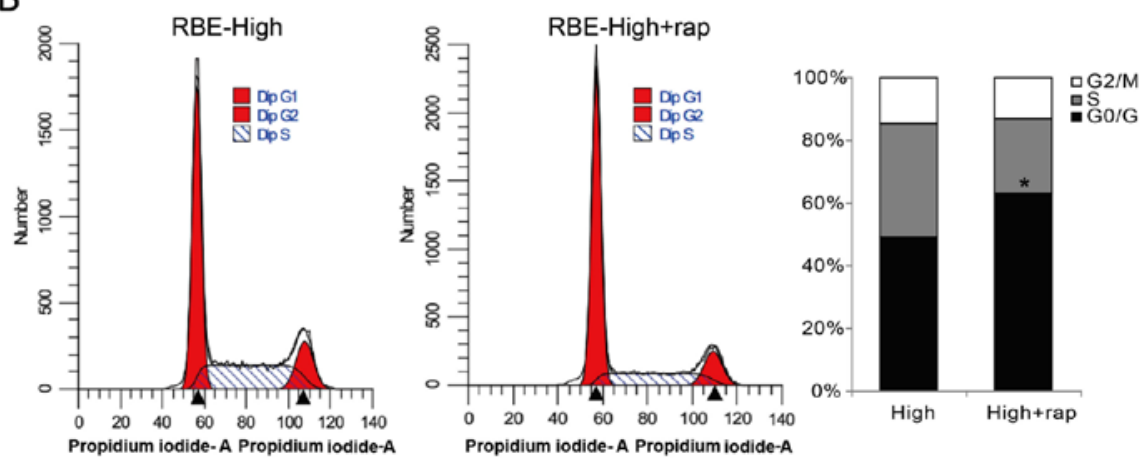

Figure 5. The mTOR pathway contributes to contact inhibition loss in human CCA cells. QBC939 (A) and RBE (B) cells at high density with or without rapamycin (rap, $100 \mathrm{nM}$ ) treatment for $24 \mathrm{~h}$ were subjected to measurement of cell cycle distribution using flow cytometry after staining with propidium iodide. Data are representative of three individual experiments. 
A



B
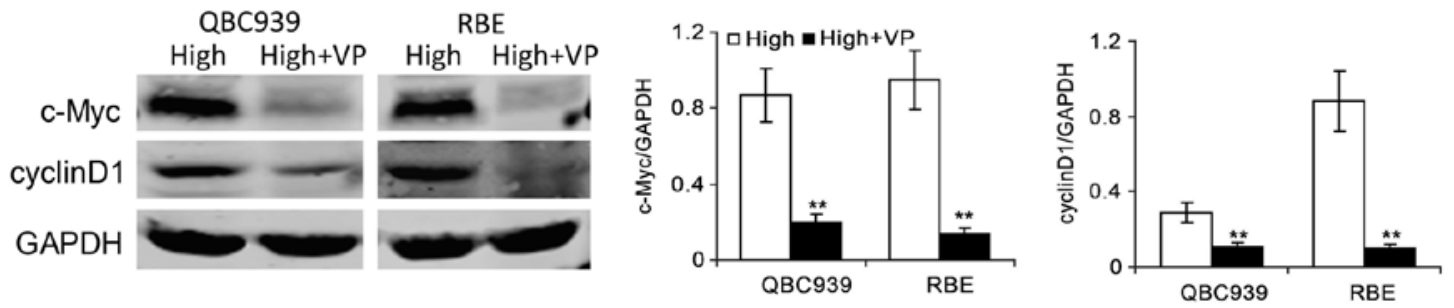

C
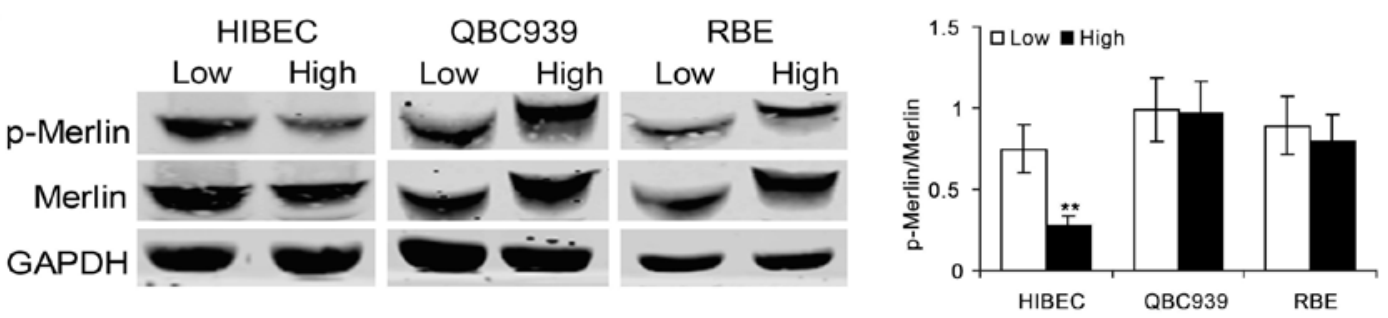

Figure 6. c-Myc mediated contact inhibition loss is regulated by YAP signaling. (A) Cell lysates from HIBEC, QBC939 and RBE cells at low and high densities were subjected to western blot analysis of p-YAP (ser127), and YAP expression. GAPDH was used as loading control. The intensities of p-YAP (ser127) were quantified by Image Studio and normalized to YAP. (B) Cell lysates from QBC939 and RBE cells at high density with or without verteporfin (VP, $10 \mu \mathrm{M}$ ) treatment for $24 \mathrm{~h}$ were subjected to western blot analysis of c-Myc and cyclin D1 expression. GAPDH was used as loading control. The intensities of c-Myc and cyclin D1 were quantified by Image Studio and normalized to GAPDH. (C) Cell lysates from HIBEC, QBC939 and RBE cells at low and high densities were subjected to western blot analysis of p-Merlin, and Merlin expression. GAPDH was used as loading control. The intensities of p-Merlin were quantified by Image Studio and normalized to Merlin.

QBC939 and RBE cells were examined. The data showed that phospho-YAP (ser127), an inactive form of YAP, markedly increased in HIBEC cells at high density (Fig. 6A), implying increased YAP protein was sequestrated in cytoplasm and was inactivated. In QBC939 and RBE cells, there was no apparent increase of the levels of phospho-YAP (ser127) at high density (Fig. 6A), indicating YAP sustains high levels of activity in confluent CCA cells. To explore whether c-Myc is regulated by YAP, we detected c-Myc protein levels after YAP inhibition by a specific inhibitor verteporfin in CCA cells. As shown in Fig. 6B, the protein levels of c-Myc significantly decreased in confluent CCA cells upon verteporfin treatment. YAP inhibition decreased cyclin D1 in confluent CCA cells. These data demonstrate that YAP is involved in c-Myc-mediated contact inhibition loss in CCA cells.

As Merlin (also named neurofibromatosis type 2 gene, NF2) is a pivotal inhibitor of YAP $(26,28)$, we investigated the activities of Merlin in HIBEC, QBC939 and RBE cells at low and high densities. The results showed that high density induced decrease of phosphor-Merlin in HIBEC cells (Fig. 6C), indicating that the upregulation of Merlin activity is required for contact inhibition in normal biliary epithelial cells. It is notable that the levels of phosphor-Merlin increased in confluent QBC939 and RBE cells (Fig. 6C), indicating that the activity of Merlin is downregulated in confluent
CCA cells. Together, these data imply a potential role of Merlin/ YAP/c-Myc signaling axis for CCA cells to override contact inhibition.

\section{Discussion}

Strict control of normal tissue size is achieved by regulating cell numbers. In normal tissues, cell number is controlled by the mechanism known as cell contact inhibition. Contact inhibition is a crucial mechanism to regulate cell proliferation in vivo and in vitro. Loss of contact inhibition is a hallmark of most human cancer cells (4). As a result, cancer cells can maintain the ability of proliferation upon reaching confluence. This criterion is also commonly used for cellular transformation in vitro (3). Consistently, we found normal biliary epithelial cells that showed apparent growth suppression at high density, while the proliferation ability of CCA cells were not apparently affected by high density. Although some important players, such as the Hippo pathway, which mediate cell cycle arrest in contact inhibition have been identified (27), the detailed molecular mechanisms mediating contact inhibition loss in cancer cells remain largely unknown.

Although the deregulation of c-Myc plays an important role in the pathogenesis of CCA (29), detailed mechanisms of how c-Myc promotes CCA remain largely unknown. Both normal 
biliary epithelial cells and human CCA cells showed strong expression of c-Myc at low density. Importantly, c-Myc clearly decreased in confluent normal biliary epithelial cells, but not in confluent CCA cells. As high density induced growth arrest of normal biliary epithelial cells, and CCA cells still sustained high ability of proliferation at high density, it is reasonable to suggest that c-Myc might be involved in contact inhibition loss in CCA cells. This speculation is supported by our data which demonstrated that the blockade of c-Myc by inhibitor 10058-F4 effectively restores contact inhibition in confluent CCA cells. Thus, c-Myc can help CCA cells to override cell-cell contact inhibition and promote the progress of CCA.

The mTOR pathway is a central pathway that regulates cell growth, survival and metabolism (30). The mTOR pathway deregulation contributes to the development and progression of most human cancers (31). Importantly, it has been reported that the deactivation of mTOR signaling is an important event for cell contact inhibition (24). Consistently, our data show that the activity of mTOR decreased obviously in contact-inhibited normal biliary epithelial cells. We and others have previously found that the mTOR pathway is implicated in the carcinogenesis and progression of CCA and mTOR inhibition suppresses the growth of human CCA cells $(25,32)$. Whether the implication of mTOR in CCA is associated with contact inhibition regulation has not been investigated. Here, our data show that CCA cells at low and high densities both display strong activity of mTOR. Based on the data that blocking mTOR by rapamycin initiated G0/G1 cell cycle arrest in confluent CCA cells, we suggest that mTOR promotes CCA cells to override contact inhibition. Importantly, we find that c-Myc suppression obviously decreased the activity of mTOR in confluent CCA cells. Thus, c-Myc promotes the loss of contact inhibition through the mTOR pathway in CCA cells. Further studies are needed to investigate the association between c-Myc and mTOR in CCA.

As the c-Myc protein decreased at confluence status in normal biliary epithelial cells but not in CCA cells, a major issue now is how c-Myc sustains high protein levels in confluent CCA cells. It has been widely reported that the oncoprotein YAP plays an essential role in cell contact inhibition and normal tissue growth control (26). Accordantly, our data show that the activity of YAP is suppressed upon high density in normal biliary epithelial cells but not in CCA cells. To figure out whether the deregulated expression of c-Myc is associated with YAP in confluent CCA cells, YAP inhibitor verteporfin, a small molecule that inhibits TEAD-YAP association, was used in our studies. The results show that verteporfin effectively decreased the protein levels of c-Myc and cyclin D1 in confluent CCA cells. Our results are consistent with the findings by Turato et al which demonstrated that knockdown YAP by siRNA reduced c-Myc proteins (33). These data demonstrated that the deregulated c-Myc in confluent CCA cells is regulated by YAP signaling. YAP is a central effector in the Hippo pathway, controlling cell-cell contact inhibition (27). It is well known that Merlin is an upstream regulator of the Hippo pathway $(27,28)$. Our data show that Merlin is involved in the deregulation of YAP in confluent CCA cells, and the deactivation of Merlin in confluent CCA cells plays an important role in YAP/c-Myc/mTOR signaling axis-mediated contact inhibition loss. It has been reported that Merlin-deficient nervous system tumors show loss of contact inhibition and activation of $\mathrm{Wnt} / \beta$ catenin and its downstream c-Myc (34). Further studies are needed to clarify whether Wnt signaling is implicated in the Merlin-YAP-c-Myc in our model, and to uncover the detailed mechanisms of Merlin deregulation in confluent CCA cells.

In conclusion, this study presents a new mechanism in which Merlin/YAP/c-Myc/mTOR signaling axis promotes human CCA cell proliferation by overriding contact inhibition. More detailed studies on the mechanism of Merlin aberrant inactivation and the link between c-Myc and mTOR in CCA cells will contribute to the understanding of molecular mechanism of cholangiocarcinogenesis and the development of new therapeutic strategies against CCA.

\section{Acknowledgements}

This study was supported by grants from the National Natural Science Foundation of China (81472312), Innovation Team of Education Department of Sichuan Province (16TD0021), Science and Technology Department of Sichuan Province Foundation (2017JY0134), Luzhou City-Southwest Medical University Foundation (2016LZXNYD-T02, 2013LZLY-J06, 2015LZCYD-S01-14/15 and 2015LZCYD-S01-8/15), Sichuan Province-Luzhou City-Southwest Medical University Foundation (14JC0082, 14JC0038 and 14ZC0070) and Education Department of Sichuan Province Foundation (15ZA0160 and 17ZA0437).

\section{References}

1. Eagle $\mathrm{H}$ and Levine EM: Growth regulatory effects of cellular interaction. Nature 213: 1102-1106, 1967.

2. Carter SB: Tissue homeostasis and the biological basis of cancer. Nature 220: 970-974, 1968.

3. Abercrombie M: Contact inhibition and malignancy. Nature 281: 259-262, 1979.

4. Hanahan D and Weinberg RA: Hallmarks of cancer: The next generation. Cell 144: 646-674, 2011.

5. Brodeur GM, Seeger RC, Schwab M, Varmus HE and Bishop JM: Amplification of $\mathrm{N}$-myc in untreated human neuroblastomas correlates with advanced disease stage. Science 224: 1121-1124, 1984.

6. Vogelstein B, Papadopoulos N, Velculescu VE, Zhou S, Diaz LA Jr and Kinzler KW: Cancer genome landscapes. Science 339: 1546-1558, 2013.

7. Kaposi-Novak P, Libbrecht L, Woo HG, Lee YH, Sears NC, Coulouarn C, Conner EA, Factor VM, Roskams T and Thorgeirsson SS: Central role of c-Myc during malignant conversion in human hepatocarcinogenesis. Cancer Res 69: 2775-2782, 2009.

8. Yang H, Liu T, Wang J, Li TW, Fan W, Peng H, Krishnan A, Gores GJ, Mato JM and Lu SC: Deregulated methionine adenosyltransferase $\alpha 1, \mathrm{c}-\mathrm{Myc}$, and Maf proteins together promote cholangiocarcinoma growth in mice and humans. Hepatology 64: 439-455, 2016.

9. Follis AV, Hammoudeh DI, Daab AT and Metallo SJ: Smallmolecule perturbation of competing interactions between c-Myc and Max. Bioorg Med Chem Lett 19: 807-810, 2009.

10. Hu J, Banerjee A and Goss DJ: Assembly of b/HLH/z proteins c-Myc, Max, and Mad1 with cognate DNA: Importance of protein-protein and protein-DNA interactions. Biochemistry 44: 11855-11863, 2005

11. Park S, Chung S, Kim KM, Jung KC, Park C, Hahm ER and Yang $\mathrm{CH}$ : Determination of binding constant of transcription factor myc-max/max-max and E-box DNA: The effect of inhibitors on the binding. Biochim Biophys Acta 1670: 217-228, 2004.

12. Dean M, Levine RA, Ran W, Kindy MS, Sonenshein GE and Campisi J: Regulation of c-myc transcription and mRNA abundance by serum growth factors and cell contact. J Biol Chem 261: 9161-9166, 1986. 
13. Reed JC, Alpers JD, Nowell PC and Hoover RG: Sequential expression of protooncogenes during lectin-stimulated mitogenesis of normal human lymphocytes. Proc Natl Acad Sci USA 83: 3982-3986, 1986.

14. Schuhmacher M and Eick D: Dose-dependent regulation of target gene expression and cell proliferation by c-Myc levels. Transcription 4: 192-197, 2013.

15. Wang H, Mannava S, Grachtchouk V, Zhuang D, Soengas MS, Gudkov AV, Prochownik EV and Nikiforov MA: c-Myc depletion inhibits proliferation of human tumor cells at various stages of the cell cycle. Oncogene 27: 1905-1915, 2008.

16. Blechacz BR and Gores GJ: Cholangiocarcinoma. Clin Liver Dis 12: 131-150, ix, 2008

17. Yang H, Li TW, Ko KS, Xia M and Lu SC: Switch from Mnt-Max to Myc-Max induces p53 and cyclin D1 expression and apoptosis during cholestasis in mouse and human hepatocytes. Hepatology 49: 860-870, 2009.

18. Yang H, Li TW, Peng J, Tang X, Ko KS, Xia M and Aller MA: A mouse model of cholestasis-associated cholangiocarcinoma and transcription factors involved in progression. Gastroenterology 141: 378-388, 388 e371-374, 2011.

19. Zhao X, Fu J, Xu A, Yu L, Zhu J, Dai R, Su B, Luo T, Li N, Qin W, et al: Gankyrin drives malignant transformation of chronic liver damage-mediated fibrosis via the Rac1/JNK pathway. Cell Death Dis 6: e1751, 2015.

20. Quelle DE, Ashmun RA, Shurtleff SA, Kato JY, Bar-Sagi D, Roussel MF and Sherr CJ: Overexpression of mouse D-type cyclins accelerates G1 phase in rodent fibroblasts. Genes Dev 7 : $1559-1571,1993$.

21. Sherr CJ and Roberts JM: CDK inhibitors: Positive and negative regulators of G1-phase progression. Genes Dev 13: 1501-1512, 1999.

22. Motti ML, Califano D, Baldassarre G, Celetti A, Merolla F, Forzati F, Napolitano M, Tavernise B, Fusco A and Viglietto G: Reduced E-cadherin expression contributes to the loss of p27kip1-mediated mechanism of contact inhibition in thyroid anaplastic carcinomas. Carcinogenesis 26: 1021-1034, 2005.

23. Bretones G, Delgado MD and León J: Myc and cell cycle control. Biochim Biophys Acta 1849: 506-516, 2015.

24. Leontieva OV, Demidenko ZN and Blagosklonny MV: Contact inhibition and high cell density deactivate the mammalian target of rapamycin pathway, thus suppressing the senescence program Proc Natl Acad Sci USA 111: 8832-8837, 2014.
25. Zhao X, Zhang C, Zhou H, Xiao B, Cheng Y, Wang J, Yao F, Duan C, Chen R, Liu Y, et al: Synergistic antitumor activity of the combination of salubrinal and rapamycin against human cholangiocarcinoma cells. Oncotarget 7: 85492-85501, 2016.

26. Zhao B, Wei X, Li W, Udan RS, Yang Q, Kim J, Xie J, Ikenoue T, $\mathrm{Yu} \mathrm{J}, \mathrm{Li} \mathrm{L}$, et al: Inactivation of YAP oncoprotein by the Hippo pathway is involved in cell contact inhibition and tissue growth control. Genes Dev 21: 2747-2761, 2007.

27. Zeng Q and Hong W: The emerging role of the hippo pathway in cell contact inhibition, organ size control, and cancer development in mammals. Cancer Cell 13: 188-192, 2008.

28. Zhang N, Bai H, David KK, Dong J, Zheng Y, Cai J, Giovannini M, Liu P, Anders RA and Pan D: The Merlin/NF2 tumor suppressor functions through the YAP oncoprotein to regulate tissue homeostasis in mammals. Dev Cell 19: 27-38, 2010.

29. Berasain C, Fernández-Barrena MG and Avila MA: New molecular interactions of c-Myc in cholangiocarcinoma may open new therapeutic opportunities. Hepatology 64: 336-339, 2016.

30. Li X, Wu C, Chen N, Gu H, Yen A, Cao L, Wang E and Wang L: $\mathrm{PI} 3 \mathrm{~K} / \mathrm{Akt} / \mathrm{mTOR}$ signaling pathway and targeted therapy for glioblastoma. Oncotarget 7: 33440-33450, 2016.

31. Kim LC, Cook RS and Chen J: mTORC1 and mTORC2 in cancer and the tumor microenvironment. Oncogene 36: 2191-2201, 2017.

32. Yothaisong S, Dokduang H, Techasen A, Namwat N, Yongvanit $P$, Bhudhisawasdi V, Puapairoj A, Riggins GJ and Loilome W: Increased activation of PI3K/AKT signaling pathway is associated with cholangiocarcinoma metastasis and PI3K/mTOR inhibition presents a possible therapeutic strategy. Tumour Biol 34: 3637-3648, 2013.

33. Turato C, Cannito S, Simonato D, Villano G, Morello E, Terrin L, Quarta S, Biasiolo A, Ruvoletto M, Martini A, et al: SerpinB3 and Yap interplay increases Myc oncogenic activity. Sci Rep 5: 17701, 2015.

34. Zhou L, Ercolano E, Ammoun S, Schmid MC, Barczyk MA and Hanemann CO: Merlin-deficient human tumors show loss of contact inhibition and activation of Wnt/ $\beta$-catenin signaling linked to the PDGFR/Src and Rac/PAK pathways. Neoplasia 13: 1101-1112, 2011. 